\title{
Adolescent Alcohol Exposure Amplifies the Incentive Value of Reward-Predictive Cues Through Potentiation of Phasic Dopamine Signaling
}

\author{
Marcia Spoelder', Kimberly T Tsutsui ${ }^{2}$, Heidi MB Lesscher', Louk JMJ Vanderschuren ${ }^{1,3}$ and Jeremy J Clark ${ }^{*, 2}$ \\ 'Department of Animals in Science and Society, Division of Behavioral Neuroscience, Faculty of Veterinary Medicine, Utrecht University, Utrecht, \\ The Netherlands; ${ }^{2}$ Department of Psychiatry and Behavioral Sciences, University of Washington, Seattle, Washington, USA; ${ }^{3}$ Department of \\ Translational Neuroscience, Brain Center Rudolf Magnus, University Medical Center Utrecht, Utrecht, The Netherlands
}

\begin{abstract}
Adolescent alcohol use remains a major public health concern due in part to well-established findings implicating the age of onset in alcohol use in the development of alcohol use disorders and persistent decision-making deficits in adults. We have previously demonstrated that moderate adolescent alcohol consumption in rats promotes suboptimal decision making and an associated perturbation in mesolimbic dopamine transmission in adulthood. Dopamine-dependent incentive learning processes are an integral component of value-based decision making and a fundamental element to many theoretical accounts of addiction. Thus we tested the hypothesis that adolescent alcohol use selectively alters incentive learning processes through perturbation of mesolimbic dopamine systems. To assess incentive learning, behavioral and neurochemical measurements were made during the acquisition, maintenance, extinction, and reacquisition of a Pavlovian conditioned approach procedure in adult rats with a history of adolescent alcohol consumption. We show that moderate adolescent alcohol consumption potentiates stimulus-evoked phasic dopamine transmission, measured in vivo by fast-scan cyclic voltammetry, in adulthood and biases individuals toward a dopamine-dependent incentive learning strategy. Moreover, we demonstrate that animals exposed to alcohol in adolescence are more sensitive to an unexpected variation in reward outcomes. This pattern of phasic dopamine signaling and the associated bias in learning may provide a mechanism for the well-documented vulnerability of individuals with early-life alcohol use for alcohol use disorders in adulthood.
\end{abstract}

Neuropsychopharmacology (20I5) 40, 2873-2885; doi: I 0.I038/npp.20I5.I39; published online 3 June 2015

\section{INTRODUCTION}

Adolescence represents a critical period of maturation in cortical and limbic brain areas involved in reward processing, inhibitory control, and decision making (Bava and Tapert 2010; Blakemore and Choudhury 2006; Chambers et al, 2003; Spear 2000). This developmental period is characterized by risky and impulsive behaviors, including experimentation with alcohol and other substances of abuse (Casey and Jones 2010). Alcohol is the most commonly used substance by adolescents and a high percentage of consumption during this period occurs in bingeing or uncontrolled use (Witt, 2010). Importantly, an increasing number of studies have revealed that the adolescent brain is particularly vulnerable to alcohol-induced functional changes (Crews et al, 2007; Guerri and Pascual 2010; Monti et al, 2005; Philpot et al, 2009; Schindler et al, 2014; Squeglia et al, 2012; Zeigler et al, 2005). Moreover, adolescent alcohol use

\footnotetext{
*Correspondence: Dr JJ Clark, Department of Psychiatry and Behavioral Sciences, University of Washington, Box 356560, 1959 NW Pacific Street, Seattle, WA 98195, USA, Tel: + I 206992 0472, Fax: + I 206 543 9520, E-mail: jjcl@uw.edu

Received I2 February 20 I5; revised I May 20 I5; accepted I May 20 I5; accepted article preview online 14 May 2015
}

increases the likelihood of developing an alcohol use disorder (AUD) in adulthood (Blomeyer et al, 2013; Dawson et al, 2008; Hingson et al, 2006).

We have previously shown that moderate adolescent alcohol consumption in rats promotes suboptimal risk preference and a corresponding increase in striatal dopamine release in response to risky choices in adulthood (Nasrallah et al, 2011). We have further demonstrated that increased risk preference may result from a selective defect in reinforcement learning (Clark et al, 2012) and that this is a specific consequence of alcohol exposure during adolescence, as identical exposure in adults does not produce this effect (Schindler et al, 2014). The mesolimbic dopamine system is implicated in reinforcement learning, goal-directed behavior, and motivational processes, including those for abused substances (Everitt and Robbins, 2005; Kelley, 2004; Robinson and Berridge, 1993; Salmone and Correa, 2012; Schultz, 2007). Alcohol, similar to virtually all abused substances, increases dopamine transmission within the ventral striatum (Cheer et al, 2007; Di Chiara and Imperato 1986; Robinson et al, 2009). Importantly, the mesolimbic dopamine system continues to mature during the adolescent period (Chambers et al, 2003), suggesting that adolescent alcohol use may alter its function, resulting in abnormal reward-related learning 
processes that impact decision making (Alaux-Cantin et al, 2013; Casey and Jones 2010; Goudriaan et al, 2007; Johnson et al, 2008; McClory and Spear 2014; Nasrallah et al, 2011; Philpot et al, 2009; Toalston et al, 2014; Zeigler et al, 2005).

Phasic dopamine transmission is evoked by salient sensory input, rewards, and predictive stimuli that have been paired with rewards during Pavlovian and instrumental conditioning (Day et al, 2007; Clark et al, 2013; Roitman et al, 2004). More recently, it has been shown that dopamine acts selectively in a form of stimulus-reward learning where incentive value is assigned to reward cues (Flagel et al, 2011). Pavlovian conditioning in a wide variety of species has been shown to elicit alternative conditioned responses where some individuals engage with the stimulus itself during cue presentation (sign trackers) and other individuals engage the site of reward delivery during cue presentation (goal trackers) (Boakes, 1977; Robinson and Flagel, 2009). The sign-tracking response is accompanied by a dynamic pattern of dopamine release in the ventral striatum, is dopamine dependent, and has been interpreted as being indicative of a learning strategy where incentive value is assigned to reward-predictive cues (Clark et al, 2012; Flagel et al, 2011). Importantly, individuals who attribute greater incentive value to reward-predictive cues during Pavlovian conditioning with natural rewards go on to exhibit greater cue-evoked motivational responses to drug-associated cues during cocaine self-administration (Yager and Robinson, 2013). Indeed, drug-associated cues exert powerful control over drug-seeking behavior, including the reinstatement of drug self-administration after extinction (Shaham et al, 2003), and individuals who assign greater incentive value to predictive cues (eg, sign trackers) are more vulnerable to this effect (Saunders and Robinson, 2010).

Here we tested the hypothesis that the behavioral and neurochemical phenotypes promoted by chronic adolescent alcohol use may contribute to the well-documented increased risk for the development of AUD through a potentiation in dopamine-mediated attribution of incentive value to rewardpaired cues. We used fast-scan cyclic voltammetry (FSCV) to measure phasic dopamine release in the nucleus accumbens core during Pavlovian conditioned approach behavior in rats that voluntarily consumed alcohol, or control gelatin, during adolescence. Dopamine release was recorded throughout the acquisition, maintenance, extinction, and reacquisition phases of the Pavlovian conditioned approach procedure. Moreover, animals were exposed to probe trials during the maintenance of Pavlovian conditioned approach behavior where better-than-expected and worse-than-expected outcomes were isolated to specifically study the effects of adolescent alcohol consumption on reward prediction error signaling. We show that moderate adolescent alcohol consumption potentiates stimulus-evoked phasic dopamine transmission in adulthood and biases individuals toward a dopamine-dependent incentive learning strategy.

\section{MATERIALS AND METHODS}

\section{Animals}

Male Sprague-Dawley rats (Charles River, Hollister, CA) obtained at postnatal day (PND) 27 were housed individually under controlled temperature and humidity conditions on a 12-h light/dark cycle (lights on at 0600 hours) with ad libitum access to water and chow (Harlan, Kent, WA). One week before the start of behavioral testing, the rats were food-restricted and maintained at $90 \%$ of their free-feeding weight. Rats were weighed and handled at least every other day throughout the course of the experiment. An outline detailing the time course of all procedures is presented in Figure 1a. All experiments were approved by the University of Washington Institutional Animal Care and Use Committee.

\section{Alcohol Administration}

Rats received 20 days of access to a $10 \%$ alcohol $(n=15)$ or control $(n=16)$ gelatin in jars in their home cage from PND 30 to 49 (Figure $1 \mathrm{~b}$ and c). The gelatin was made available $24 \mathrm{~h}$ /day in addition to ad libitum water and chow, and the jars were replaced with fresh gelatin every day. Alcohol and control gelatin was prepared as previously described (Clark et al, 2012; Nasrallah et al, 2009). Upon completion of the 20-day alcohol and control exposure, jars were removed and the rats were monitored daily for withdrawal symptoms. Systematic measurements of intoxication and withdrawal symptoms were not made in these animals but no overt signs of withdrawal symptoms were observed (eg, seizures, weight loss, and anxious behavior during handling). In addition, blood ethanol concentration (BEC) measurements were not made as it is challenging to get an accurate measure of peak BEC with voluntary, free access models as we have no control over when the animals consume the gelatin. Therefore, any measure of BEC is likely to be a systematic underestimate because the average $\mathrm{BEC}$ will include animals that do not consume alcohol during the sampling period chosen. However, we have previously examined BEC after alcohol gelatin exposure during adolescence (Schindler et al, 2014) as have others (Rowland et al, 2005; McMurray et al, 2014). To assess BEC, we isolated peak intake periods to get an accurate estimate given a specific amount of intake. We observed an average BEC of $35 \mathrm{mg} \%$ (the BEC levels ranged between 10 and $80 \mathrm{mg} \%$ ), and the BEC values were highly correlated with alcohol intake. Daily alcohol intake in the current experiments averaged $11.5 \mathrm{~g} / \mathrm{kg}$. This is a substantial amount of alcohol in comparison to other free-access models and is consistent with the intake from our BEC studies.

\section{Surgery and Electrochemical Detection of Dopamine}

One week after cessation of alcohol access, rats were implanted with bilateral carbon-fiber microelectrodes targeting the nucleus accumbens core $(1.3 \mathrm{~mm}$ lateral, $1.3 \mathrm{~mm}$ rostral, and $6.8 \mathrm{~mm}$ ventral of bregma) for in vivo detection of phasic dopamine using FSCV as previously described (Clark et al, 2010). Of the 31 rats, 5 were excluded from the voltammetry data analyses: 1 had electrode placements outside the nucleus accumbens core, 3 lost headcaps over the course of the experiment, and 1 did not have reliable recordings. Rats were placed in an operant chamber (see below) and connected to a head-mounted voltammetric amplifier. Waveform generation, data acquisition, and analysis were carried out on a PC-based system using two PCI multifunction data acquisition cards and software written in LabVIEW (National Instruments). Reward- 
a

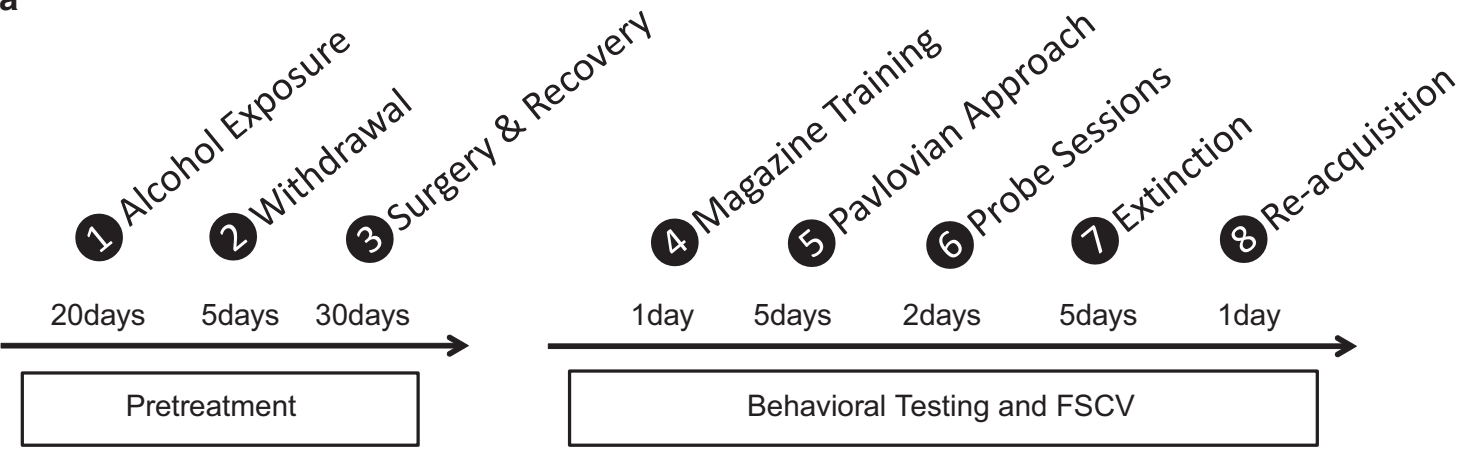

b



C

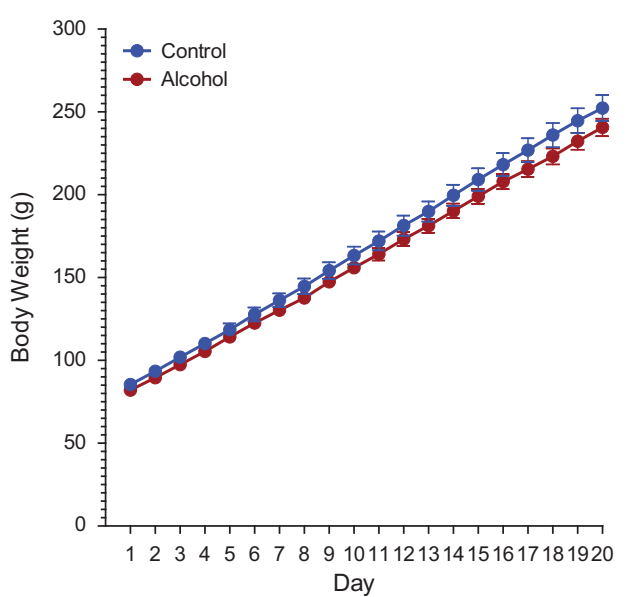

d

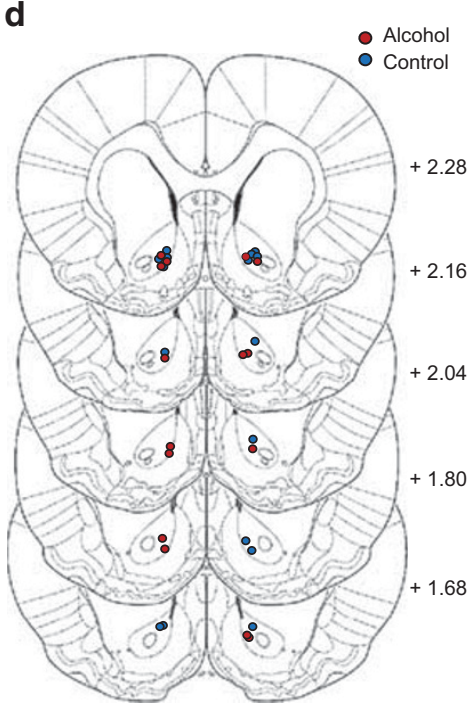

Figure I (a) Procedural timeline of the experiments. (b) Adolescent alcohol consumption during PND 30-49 was stable across the 20-day continuous exposure period. (c) Animals exposed to either control or alcohol gelatin increased in body weight to a similar extent. (d) Coronal sections of the rat brain showing the recording sites in the nucleus accumbens core (Adapted from Paxinos and Watson, 2004).

evoked dopamine release in response to uncued sucrose pellet delivery was used to ensure electrode viability prior to each behavioral session. Dopamine was isolated from the voltammetric signal with chemometric analysis (Heien et al, 2005) using a standard training set based on stimulated dopamine release. Dopamine concentration was estimated based on the average postimplantation electrode sensitivity (Clark et al, 2010). Peak CS- and US-evoked dopamine values were obtained by taking the largest value in the 3-s period after stimulus presentation.

\section{Apparatus}

Equipment and procedures for Pavlovian conditioning have been described in detail elsewhere (Flagel et al, 2011). Briefly, the rats were trained and tested in operant conditioning chambers (Med Associates, St Albans, VT) situated in soundattenuating cubicles. Each chamber was equipped with two retractable levers and a food cup within a recessed magazine situated between the levers. A cue light was present above each lever, and a house light was located on the opposite wall. Sucrose pellets ( $45 \mathrm{mg}$, Bio Serve) were delivered in the food cup via a dispenser. Experimental events and data recording were controlled using the MED-PC software for Windows (Med Associates).

\section{Behavioral Procedures}

Rats received sucrose pellets in their home cage for 2 days before training to reduce potential food neophobia. All behavioral sessions were conducted between 1000 and 1900 hours. The rats were habituated to the operant chamber for one session during which 15 sucrose pellets were randomly delivered over the course of $15 \mathrm{~min}$. The Pavlovian conditioned approach procedure was conducted as previously described (Flagel et al, 2011). Briefly, a trial consisted of the insertion of the left or right lever (counterbalanced between rats) and the illumination of a cue light above the designated lever (conditioned stimulus, CS) for $8 \mathrm{~s}$, followed by the immediate delivery of two sucrose pellets (unconditioned stimulus, US) and the illumination of the light in the recessed magazine. Twenty-five CS-US presentations occurred on a variable inter-trial interval from a range of values $(30,40,50,60,70,80$, and $90 \mathrm{~s})$ in each session. Lever presses and food cup entries during lever presentation were recorded but had no programmed consequences. After 5 Pavlovian conditioned approach sessions, the rats were given two probe 
sessions consisting of a pseudorandom presentation of different reward sizes $(0,1,2,3$, or 4 sucrose pellets; 5 trials of each reward size), separated by a standard Pavlovian conditioned approach session with 2 sucrose pellets. The rats then received five extinction sessions in which the trial structure remained the same except that CS presentation was not followed by reward delivery. Finally, the rats received a Pavlovian conditioned approach session to assess reacquisition.

\section{Histological Verification of Recording Sites}

At the end of experimentation, rats were anesthetized with sodium pentobarbital, and recording sites were marked with an electrolytic lesion $(300 \mathrm{~V})$ by applying current directly through the recording electrode for $20 \mathrm{~s}$. Rats were then transcardially perfused with PBS followed by $4 \%$ paraformaldehyde. The brains were removed and kept in $4 \%$ paraformaldehyde followed by 15 and $30 \%$ sucrose solution at $4{ }^{\circ} \mathrm{C}$, each for 1-2 days, and then rapidly frozen in aluminum foil on dry ice and stored at $-20^{\circ} \mathrm{C}$. Brains were sliced on a cryostat $\left(50-\mu \mathrm{m}\right.$ coronal sections, $\left.-20^{\circ} \mathrm{C}\right)$ and stained with cresyl violet to aid in visualization of anatomical structures. Electrode locations were confirmed to be in the core of the nucleus accumbens (Figure 1d).

\section{Data Analysis}

All statistical analyses were conducted using SPSS 20.0 for Windows and GraphPad Prism 6. Behavioral and voltammetry data were binned into five-trial epochs. A response bias score, ie, a measure of the relative allocation of behavioral responses, was calculated by subtracting the number of magazine entries from the number of lever presses divided by the sum of both responses: (lever presses-magazine entries)/(lever presses+magazine entries), resulting in a number ranging from -1 (goal-tracking response) to +1 (sign-tracking response) (Meyer et al, 2012). Based on previous work, animals with a response bias $>+0.70$ were defined as animals with a strong sign-tracking bias (Flagel et al, 2008; Meyer et al, 2012) and were used for additional neurochemical analyses. Conditioned responses from all phases of training were analyzed using linear mixed-effects models (Verbeke and Molenberghs, 2000) in which treatment group (alcohol and control) and trial bin were treated as independent variables. Each parameter and the residuals of the behavioral parameters used in the linear-effects model were tested for normality with a Kolmogorov-Smirnov test. For all analyses, the covariance structure was explored and modeled appropriately. When significant main effects or interactions were detected, Bonferroni post-hoc comparisons were made. Statistical analyses of the voltammetry data were performed using one-, two-, and three way repeatedmeasures ANOVAs, with peak dopamine values upon CS and US presentation, reward size, or trial bin as withinsubject variables and group (alcohol or control) as betweensubject variable. In case of significant main effects in the voltammetry data, post-hoc analyses were performed using pairwise comparisons with a Bonferroni correction. The threshold for statistical significance was set at $p<0.05$. All data are presented as mean \pm SEM. Graphs were made using Graphpad Prism 6.

\section{RESULTS}

\section{Alcohol Intake}

Daily adolescent alcohol intake was stable across the 20-day exposure period $\left(\mathrm{F}_{(19,266)}\right.$ day $\left.=1.62, \mathrm{NS}\right)$ and averaged $11.5 \pm 0.98 \mathrm{~g} / \mathrm{kg}$, comparable to our previous studies (Figure 1b; Nasrallah et al, 2011; Schindler et al, 2014). The caloric intake was comparable for alcohol- and controlexposed animals $\left(\mathrm{F}_{(6,172)}\right.$ day $\times$ group $=1.69, \mathrm{NS} ; \mathrm{F}_{(1,29) \text { group }}=$ $0.184, \mathrm{NS}$ ) and both groups increased in body weight to the same extent over the course of the 20-day exposure period

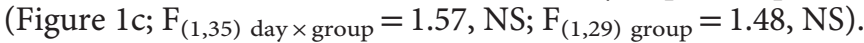

\section{Acquisition of Pavlovian Conditioned Approach Behavior After Adolescent Alcohol Exposure}

Pavlovian conditioned approach responses to either the reward-predicting lever (sign tracking) or the food magazine (goal tracking) during CS presentation developed differentially for the alcohol-exposed and control groups over the course of training (Figure 2). The response bias developed over trial bins toward a sign-tracking response in both groups $\left(\mathrm{F}_{(24,89)}\right.$ trial bin $\left.=18.81, p<0.001\right)$; however, the alcohol-exposed animals showed a significantly stronger sign-tracking bias relative to control animals (Figure 2a; $\mathrm{F}_{(24,89)}$ trial bin $\times$ group $=2.13, p<0.01, \mathrm{~F}_{(1,49)}$ group $=4.35$, $p<0.05)$. Indeed, control animals showed a conditioned response (CR) towards the food magazine or the lever, or both, whereas the distribution of approach behavior in animals treated with alcohol during adolescence was shifted exclusively towards sign-tracking CRs (Figure 2b; Levene's Test: $\mathrm{F}=12.47, p<0.05)$. The $\mathrm{CR}$ towards the food magazine decreased over trials in alcohol-exposed animals, whereas it remained at the same level for control animals (Figure 2c; $\mathrm{F}_{(24,221)}$ trial bin $=5.84, p<0.001 ; \mathrm{F}_{(1,48) \text { group }}=6.24, p<0.05$; $\mathrm{F}_{(24,221)}$ trial bin $\times$ group $\left.=2.37, p<0.01\right)$. The number of lever contacts upon cue presentation increased in both groups during learning (Figure $2 \mathrm{~d} ; \mathrm{F}_{(24,103)}$ trial bin $=9.15, p<0.001$ ). This overall pattern of behavior and statistical results was unchanged after exclusion of the animals without viable electrodes for FSCV (data not shown).

\section{Stimulus-Evoked Phasic Dopamine Signaling During Acquisition}

Phasic dopamine release was evoked by both CS and US presentation during early acquisition (first 25 trials, session 1) in both groups as previously described (Clark et al, 2013; Day et al, 2007; Flagel et al, 2011). However, phasic dopamine transmission was significantly higher overall in animals with a history of adolescent alcohol exposure during this phase of learning (Figure $3 \mathrm{a}-\mathrm{c} ; \mathrm{F}_{(1,58)}$ group $=5.49$, $p<0.05)$. Analysis of phasic dopamine release to rewardrelated stimuli across all trial bins revealed that phasic dopamine release developed differentially in response to CS and US presentation $\left(\mathrm{F}_{(15,426) \text { stimulus } \times \text { trial bin }}=10.42, p<0.001\right)$ independent of treatment $\left(\mathrm{F}_{(15,426)}\right.$ stimulus $\times$ trial bin $\times$ group $=$ 1.34, NS). Previous studies have shown that the pattern of phasic dopamine release evoked by CS and US presentation during learning is linked to the behavioral responses toward reward-related stimuli and the attribution of incentive value to predictive cues (Flagel et al, 2011). Because behavioral 
a

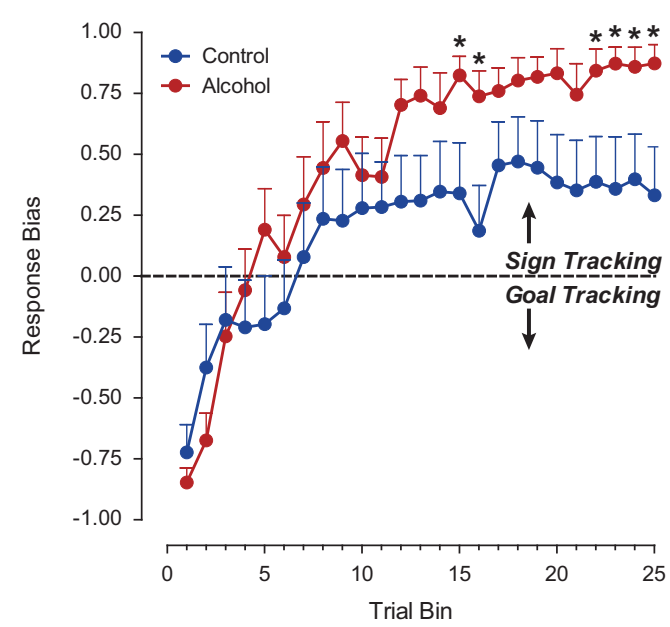

c

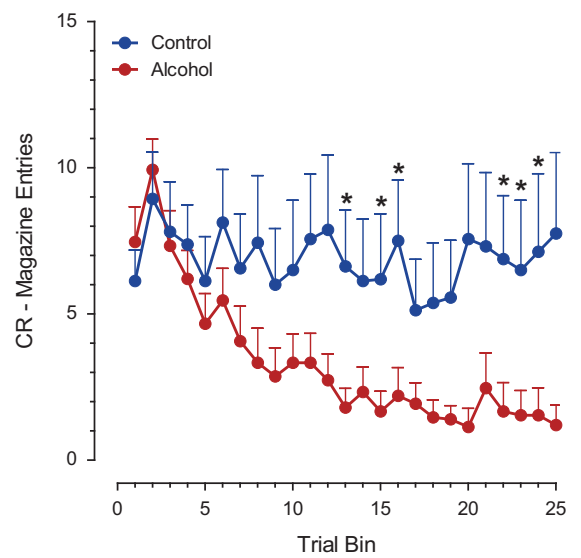

b



d



Figure 2 Behavioral responses during the Pavlovian conditioned approach task. (a) Analysis of response bias (lever presses - food cup entries)/(lever presses+food cup entries), a measure of the relative allocation of behavioral responses, revealed that animals exposed to alcohol during adolescence mainly show CRs to the reward-predictive cue. (b) A frequency distribution of response bias scores during the last session of training indicates that animals exposed to alcohol during adolescence shifted the distribution of responses exclusively towards a sign-tracking CR. (c) Over the course of learning, alcohol-exposed animals reduced their CR towards the food cup, whereas control-treated animals continued to approach the food cup. (d) CRs to the reward-predicting lever increased in both groups over training. Data are represented as means+SEM. *Indicates significant difference between groups with post-hoc $t$-tests with a Bonferroni correction $(p<0.05)$

responses directed toward either the predictive cue (sign tracking) or reward location (goal tracking) may reflect different learning mechanisms (Clark et al, 2012), we performed a similar analysis of phasic dopamine transmission in animals with a strong sign-tracking bias (defined as response bias $>+0.70$; Figure $4 a$ ) over the course of learning. Importantly, phasic dopamine release was further increased in sign-tracking animals with a history of alcohol exposure in comparison to controls $\left(\mathrm{F}_{(1.23) \text { roup }}=8.71, p<0.05\right)$ (Figure $4 \mathrm{~b}$ and $c$ ), including higher CS-evoked dopamine release throughout learning $\left(\mathrm{F}_{(1,23)}\right.$ group $\left.=6.15, p<0.05\right)$. Significantly higher CS-evoked dopamine release in animals with a more extreme sign-tracking phenotype after alcohol exposure is consistent with our previous findings where animals selected for extreme phenotypes differed in CS-evoked dopamine release but animals with mixed behavioral responses did not. These data indicate that alcohol exposure further enhances CS-evoked dopamine release in sign trackers, possibly resulting in a stronger bias toward a dopamine-dependent incentive-learning strategy.
Separate analysis of CS- and US-evoked dopamine release in all animals revealed that CS-evoked dopamine release increased during the first trial bins of the Pavlovian conditioning sessions $2-5\left(\mathrm{~F}_{(11,326)}\right.$ trial bin $\left.=11.88, p<0.05\right)$, whereas US-evoked phasic dopamine release steadily decreased during learning $\left(\mathrm{F}_{(10,290)}\right.$ trial bin $\left.=13.26, p<0.05\right)$. This profile is consistent with the view that phasic dopamine transmission encodes a reward prediction error of the type used as a teaching signal in formal models of reinforcement learning (Schultz et al, 1997). Dopamine transmission after behavior had reached asymptote (last 25 trials, session 5) was comparable between the treatment groups in response to both the CS and US presentation (Figure 3d).

\section{Extinction of Sign-Tracking and Goal-Tracking Responses}

During extinction, all animals reduced their conditioned approach behavior. The response bias score decreased across trials equally in both groups and fluctuated around zero by 

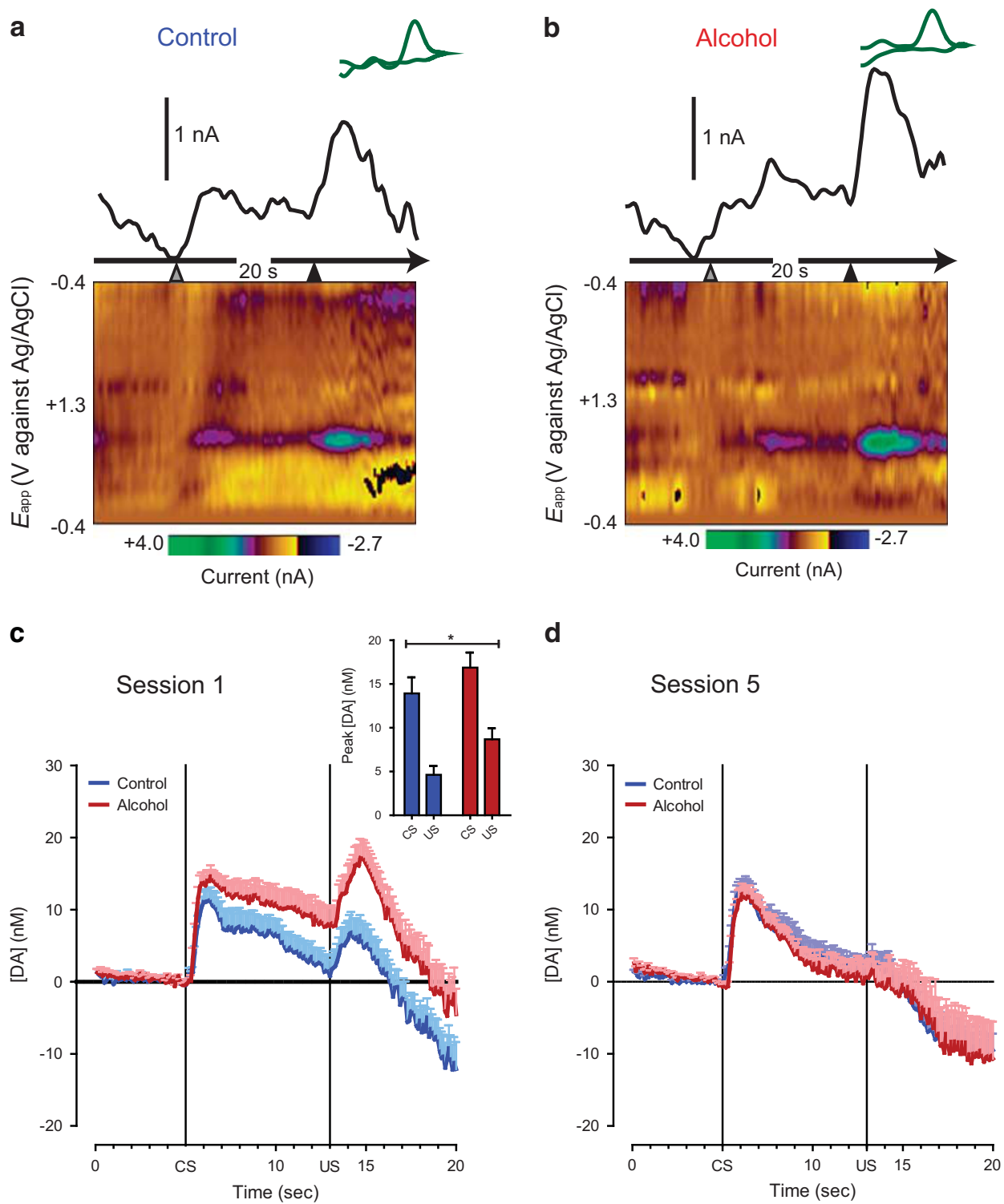

Figure 3 Phasic dopamine signaling during the first and final sessions of Pavlovian conditioned approach behavior. (a, b) Representative traces from the first session and corresponding background-subtracted cyclic voltammograms (inset) depict changes in dopamine oxidative current within the nucleus accumbens core in response to CS presentation (grey arrowhead) after $5 \mathrm{~s}$ and US delivery (black arrowhead) after I $3 \mathrm{~s}$ in control (a) and alcohol-exposed animals (b). The pseudocolor plots depict color-coded observed changes in redox currents as a function of applied potential ( $y$ axis) plotted over time ( $x$ axis). (c) Average trace of dopamine transmission in a 20-s window around CS and US presentation over the first 25 trials of Pavlovian conditioning. (c: inset) Peak dopamine values for CS and US responses for alcohol- and control-treated animals in the first Pavlovian session. (d) Average trace of dopamine transmission in a 20-s window around CS and US presentation over the final 25 trials of Pavlovian conditioning. Data are represented as means+SEM. *Difference between groups (one-way ANOVA, $p<0.05$ ).

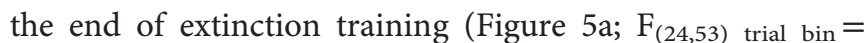
27.77, $p<0.001)$. Analysis of the food cup-directed CR revealed a main effect of extinction training (Figure $5 b$; $\left.\mathrm{F}_{(24,102) \text { trial bin }}=1.67, p<0.05\right)$ and a main effect of treatment group $\left(\mathrm{F}_{(1,98) \text { group }}=6.77, p<0.05\right)$. The total number of lever contacts upon cue presentation decreased in both groups across trials (Figure $5 \mathrm{c} ; \mathrm{F}_{(24,47)}$ trial bin $=25.91, p<0.001$ ). Consistent with the behavioral data, CS-evoked dopamine release decreased across the extinction phase (Figure $5 \mathrm{~d}$; $\left.\mathrm{F}_{(9,246) \text { trial bin }}=22.17, p<0.001\right)$, and this was not different between groups $\left(\mathrm{F}_{(9,246)}\right.$ trial bin $\times$ group $\left.=0.92, \mathrm{NS}\right)$.

\section{Reacquisition of Sign-Tracking and Goal-Tracking Conditioned Responses}

After extinction training, the animals were exposed to a final conditioning session in which they were again rewarded with two sucrose pellets upon lever retraction to assess reacquisition of Pavlovian conditioned approach behavior. In order to analyze the change in behavior from extinction baseline through reacquisition, we performed a repeated measure analyses over the last five trial bins of extinction and the five trial bins of reacquisition (Figure $6 \mathrm{a}-\mathrm{c}$ ). Consistent with 


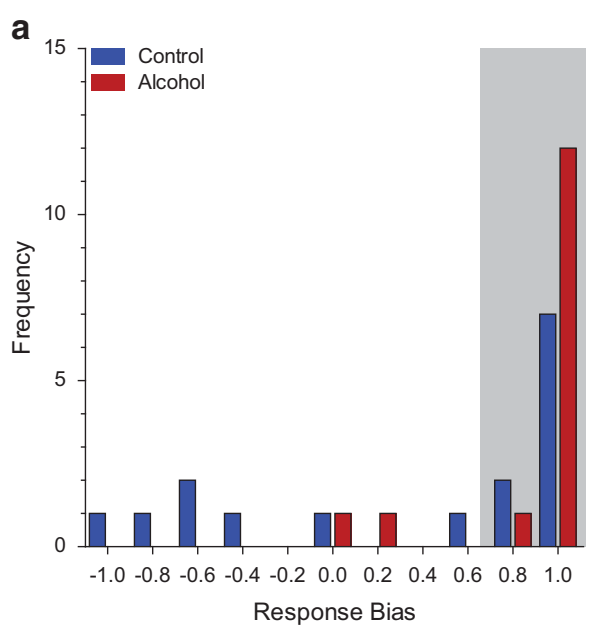

b


Figure 4 CS- and US-evoked phasic dopamine signaling across training in animals classified as sign trackers. (a) A frequency distribution of response bias scores during the last session of training. The gray bar indicates animals with a response bias score $>0.70$ that were included in voltammetric analyses in panels (b) and (c). (b) CS-evoked dopamine in the alcohol and control groups throughout training. (c) US-evoked dopamine in the alcohol and control groups throughout training. *Indicates significant difference between groups with post-hoc $t$-tests with a Bonferroni correction $(p<0.05)$.

acquisition, this analysis revealed that the conditioned response developed differently for the two treatment groups during reacquisition, as alcohol-exposed animals showed a greater bias toward a sign-tracking response. This was confirmed by analysis of the response bias, which revealed a main effect of trial bins (Figure $6 \mathrm{a} ; \mathrm{F}_{(9,225)}$ trial bin $=19.24$, $p<0.001)$ and a significant interaction between trial bins and group $\left(\mathrm{F}_{(9,225)}\right.$ trial bin $\times$ group $\left.=2.25, p<0.05\right)$. Control animals reacquired their approach behavior toward the food cup during this phase as indicated by a main effect of trial bins $\left(\mathrm{F}_{(9,61)}\right.$ trial bin $\left.=4.79, p<0.001\right)$ and did so to a greater extent than alcohol-treated animals as indicted by a main effect of treatment (Figure $6 \mathrm{~b} ; \mathrm{F}_{(9,61)}$ group $=5.46, p<0.05$ ). The total number of lever contacts upon cue presentation increased in both groups (Figure $6 c ; \mathrm{F}_{(9,33)}$ trial bin $=19.19, p<0.001$ ).

\section{Stimulus-Evoked Dopamine Signaling During Reacquisition}

CS-evoked (Figure 6d) and US-evoked (Figure 6e) phasic dopamine signaling during the reacquisition session increased above extinction baseline in both groups $\left(\mathrm{CS} ; \mathrm{F}_{(9,270) \text { trial bin }=}\right.$ 22.83, $p<0.001$ : US; $\mathrm{F}_{(9,270)}$ trial bin $\left.=10.64 p<0.001\right)$ in parallel with the reacquisition of conditioned behavioral responding. Consistent with the increased response bias score in alcohol-exposed animals, phasic dopamine transmission in alcohol-exposed animals was significantly higher for both stimuli in comparison to controls $\left(\mathrm{CS} ; \mathrm{F}_{(1,270) \text { group }}=\right.$ 4.59, $p<0.05$ : US; $\left.\mathrm{F}_{(1,270) \text { group }}=16.56, p<0.001\right)$.

\section{Dopamine Encoding of Positive and Negative Reward Prediction Errors}

Consistent with phasic dopamine encoding for positive and negative reward prediction errors, phasic dopamine release after unexpected reward presentation and omission was sensitive to variation in reward size (Figure $7 \mathrm{a}-\mathrm{d} ; \mathrm{F}_{(2,54)}=9.23$, $p<0.001)$. Moreover, the dopaminergic response to the variation in reward sizes was different upon CS or US presentation $\left(\mathrm{F}_{(2,54) \text { reward size } \times \text { stimulus }}=4.15, p<0.05\right)$, indicating that the US response was affected by the reward size (Figure $7 \mathrm{~d}$; $\left.\mathrm{F}_{(2,48)}=15.07, p<0.001\right)$, whereas the CS responses remained unaltered (Figure $7 \mathrm{c} ; \mathrm{F}_{(2,54)}=0.55$, NS). Interestingly, alcoholtreated animals showed greater overall responsiveness to positive prediction errors $\left(\mathrm{F}_{(2,48)}\right.$ reward size US $\times$ group $=4.03$, $p<0.05)$, which is in line with the neurochemical and behavioral data from both acquisition and reacquisition. Post-hoc analyses indicated that alcohol-exposed animals showed a higher dopamine release upon better-thanexpected rewards in comparison to both the neutral and worse-than-expected rewards $(p<0.004)$, whereas the dopamine release upon the better-than-expected reward in the control animals was only higher in comparison to the worsethan-expected reward $(p<0.01$; Figure $7 d)$. 
a



b

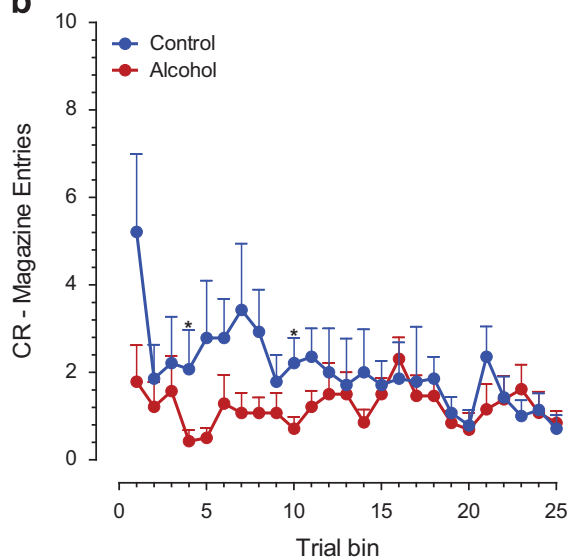

c

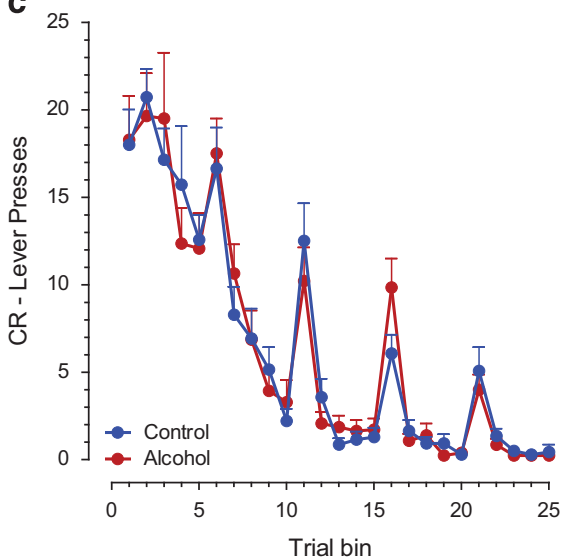

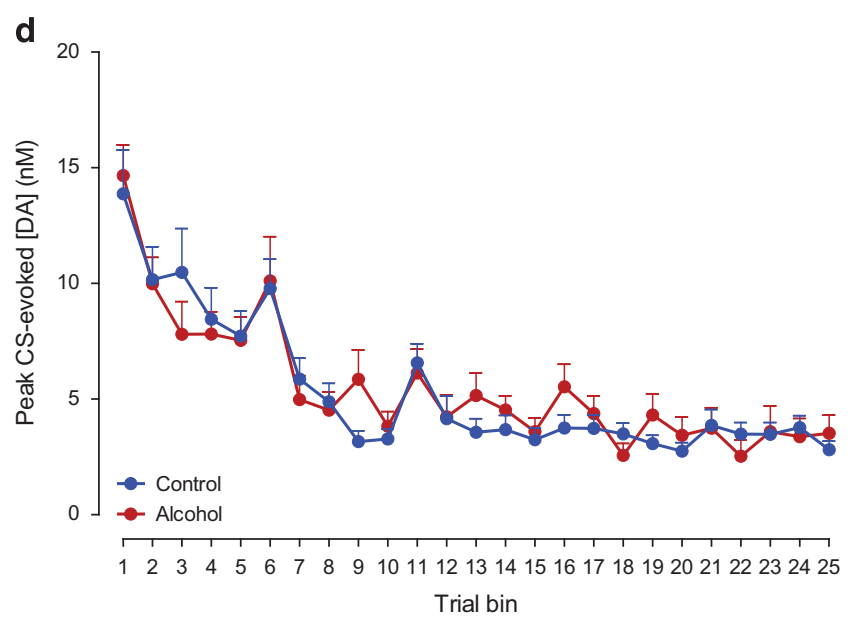

Figure 5 Behavioral responses and CS-evoked phasic dopamine signaling during extinction training. (a) The response bias score decreased equally in both groups. (b) The food cup-directed CR declined in control animals, whereas it remained unchanged for alcohol-exposed animals. (c) Conditioned responses towards the reward-predictive lever decreased across trials in both the groups. (d) CS-evoked phasic dopamine decreased across extinction equally in both groups. *Indicates significant difference between groups with post-hoc $t$-tests with a Bonferroni correction $(p<0.05)$.

\section{DISCUSSION}

To examine a potential mechanism by which adolescent alcohol consumption increases the vulnerability to AUD in adulthood, we investigated phasic dopamine signaling in the nucleus accumbens core during Pavlovian conditioned approach behavior in adult rats that had voluntarily consumed alcohol during adolescence. We report that moderate alcohol consumption during adolescence increases the assignment of incentive value to reward-predictive cues in adulthood. This perturbation in incentive learning processes was associated with a potentiation of stimulus-evoked phasic dopamine transmission during early acquisition as well as during reacquisition of Pavlovian conditioned approach behavior. Importantly, when analysis was restricted to sign-tracking animals, CS-evoked dopamine release was significantly elevated by prior alcohol exposure. Moreover, during probe trials, alcohol-exposed animals showed a heightened sensitivity to outcomes that were better than expected as evidenced by greater phasic dopamine signaling to positive prediction errors. Thus, our results indicate that adolescent alcohol exposure promotes long-lasting alterations in dopaminedependent incentive learning.
Pavlovian conditioning processes are an important contributor to addictive behaviors as substance-associated cues can drive drug craving, drug seeking, and promote relapse following abstinence (Milton and Everitt, 2012; O'Brien et al, 1998; Shaham et al, 2003; Stewart et al, 1984). Indeed, individuals who abuse drugs are more likely to exhibit this stimulus-driven affective behavior (Bickel and Marsch, 2001). The sign-tracking phenotype in rats, characterized by the assignment of incentive value to reward-predictive cues, has been previously associated with reduced impulse control and vulnerability to addictive behavior (Flagel et al, 2010; Lovic et al, 2011; Tomie et al, 2008). Importantly, there is a wide variation in the degree to which individuals engage in sign-tracking behavior, thus allowing for a comparison between drug-associated behaviors and the degree to which individuals assign incentive value to predictive cues. Multiple reports have shown that Pavlovian cues associated with the delivery of drugs of abuse acquire greater control over motivated behavior in animals selected for sign-tracking behavior (Saunders et al, 2013; Yager and Robinson, 2013; Yager et al, 2014). This suggests that these animals are more vulnerable to the influence of reward-predicting stimuli, a characteristic which is associated with compulsive and 


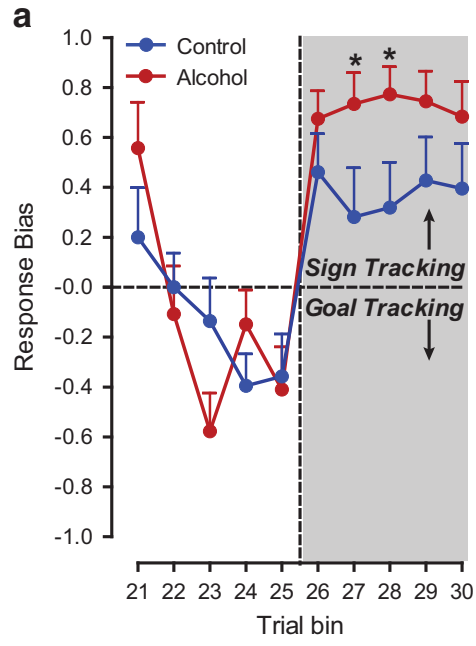

b

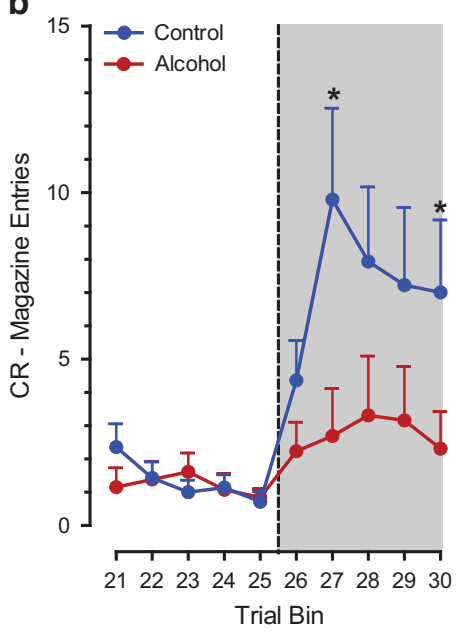

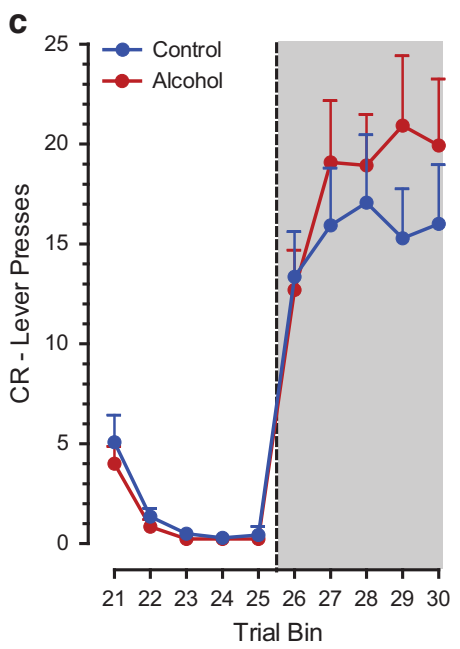
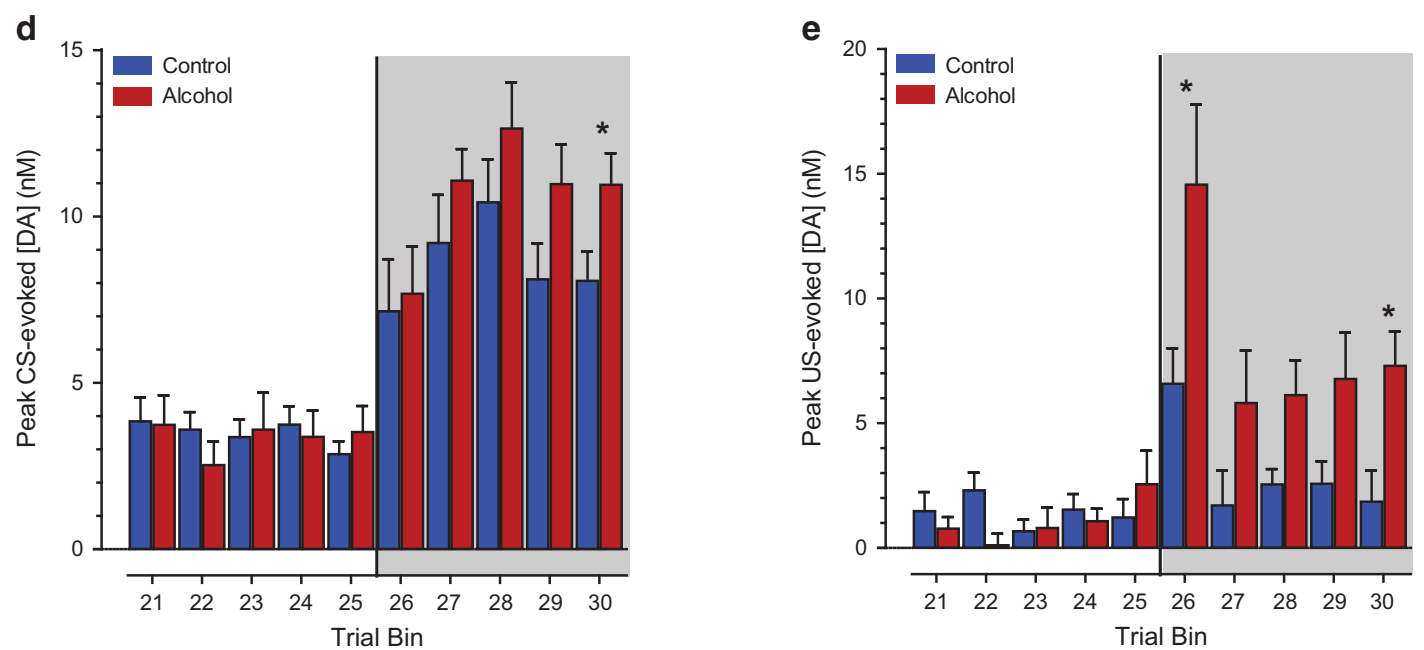

Figure 6 Behavioral $(a-c)$ and dopaminergic responses ( $d, e)$ during reacquisition of Pavlovian conditioned approach behavior. (a) Alcohol-exposed animals showed a greater bias toward a sign-tracking response. (b) The CR toward the food cup mainly increased in control animals, whereas (c) both groups increased their CR for the reward-predicting lever. (d, e) CS- and US-evoked phasic dopamine signaling increased during reacquisition in both groups. (d) Adolescent alcohol consumption resulted in higher CS-evoked phasic dopamine release in the final trial bin of reacquisition in comparison to controls, as well as a (e) larger US-evoked phasic dopamine release in the first trial bin. Subsequently, in trial bins 2-5, both groups showed a decrease in US-evoked dopamine release, but signaling remained higher in alcohol-exposed animals. *Indicates significant difference between groups with post-hoc t-tests with a Bonferroni correction $(p<0.05)$.

relapsing drug abuse (Milton and Everitt, 2012; O'Brien et al, 1998; Shaham et al, 2003; Stewart et al, 1984). Here we demonstrate that adolescent alcohol exposure shifts the normal distribution of conditioned responses elicited by Pavlovian cues exclusively toward a sign-tracking phenotype. These data are in support of a previous study which reported that alcohol exposure during adolescence, but not during adulthood, increased sign-tracking behavior in rats (McClory and Spear, 2014). Indeed, the sign-tracking phenotype has been specifically linked to models of AUD (Tomie and Sharma, 2013). Behavioral responses during extinction were similar between groups, replicating our previous findings and supporting the view that adolescent alcohol exposure produces over-fast learning for better-than expected but not worse-than-expected outcomes (Clark et al, 2012).

The core subregion of nucleus accumbens is implicated in the acquisition and maintenance of Pavlovian conditioned approach behavior (Di Ciano et al, 2001; Parkinson et al,
2002), and dopamine transmission in this structure is evoked by rewards and reward-predictive cues (Clark et al, 2013; Day et al, 2007). This pattern of phasic dopamine release is linked to the behavioral responses elicited by reward-related stimuli where sign-tracking animals show this pattern and goal-tracking animals do not (Flagel et al, 2011). Indeed, the role of dopamine signaling in sign-tracking behavior has been extensively studied in previous work (Di Ciano et al, 2001; Flagel et al, 2011; Saunders and Robinson, 2012; Clark et al, 2013). These studies have shown that systemic and intracranial infusions of the dopamine D1/D2 antagonist flupenthixol into the nucleus accumbens core reduced sign-tracking behavior during both the acquisition and performance of Pavlovian conditioned approach behavior. Moreover, it was shown that dopamine is necessary for the learning of a sign-tracking conditioned response, whereas it is not necessary for learning a goal-tracking conditioned response (Flagel et al, 2011). In the current study, adult 


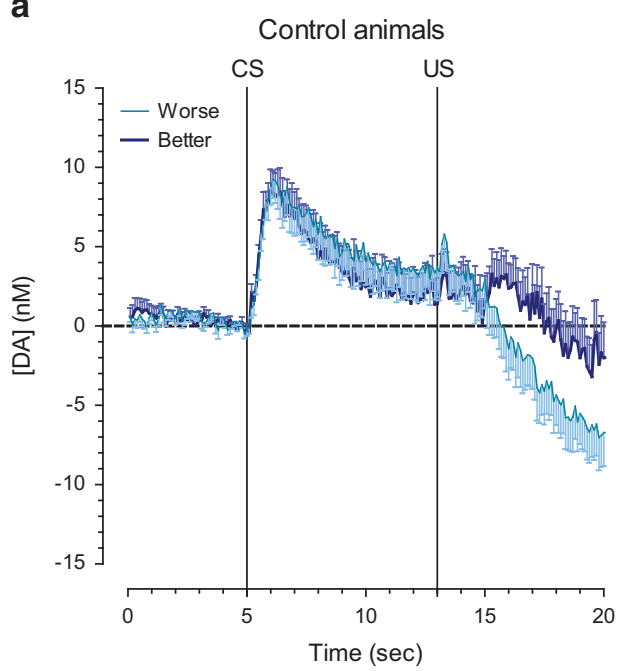

C

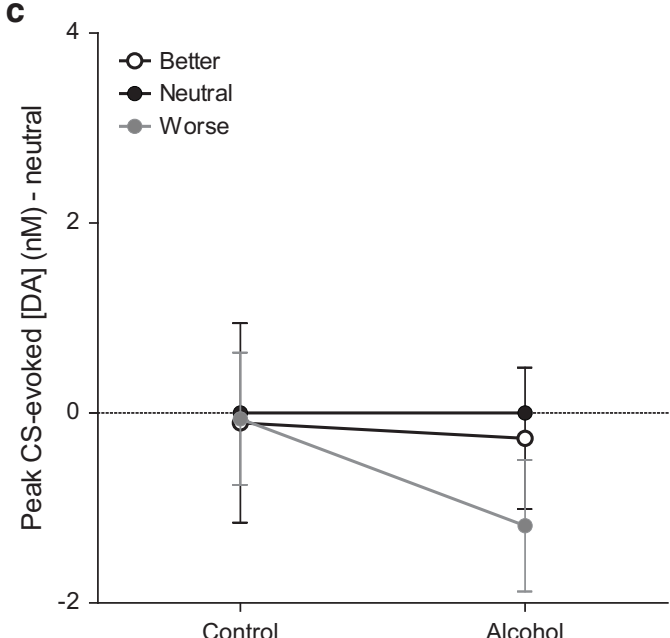

b

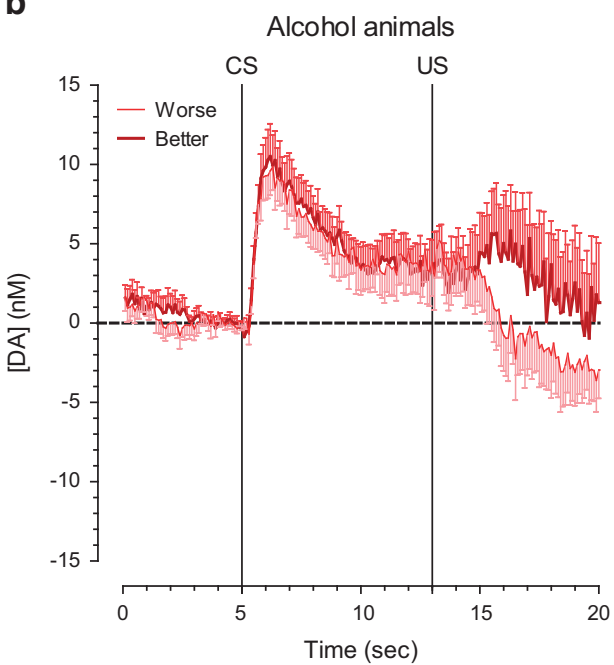

d



Figure 7 Phasic dopamine signaling in response to worse-than-expected, expected, and better-than-expected reward outcomes in control- and alcoholtreated animals. (a, b) Average dopamine traces for worse- (reward sizes 0 and I) and better-than-expected (reward sizes 3 and 4 ) outcomes during the probe sessions where reward size was varied unpredictably. (c) CS-evoked dopamine release was not affected by altered reward sizes. (d) US-evoked dopamine release was sensitive to varying reward size in both groups but alcohol-treated animals showed greater overall responsiveness to unexpected variation in reward outcomes. *Indicates significant difference in US dopamine release between reward sizes in post hoc within group comparisons with a Bonferroni correction $(p<0.05)$.

animals exposed to alcohol during adolescence showed enhanced phasic dopamine release during early acquisition of Pavlovian conditioned approach behavior preceding the development of the bias toward a sign-tracking phenotype. Because the behavioral response to either the rewardpredicting lever (sign tracking) or the reward location (goal tracking) may reflect different learning mechanisms (Clark et $a l, 2012$ ), we also examined phasic dopamine transmission exclusively in sign-tracking animals from both groups over the course of learning. These analyses revealed that alcoholtreated sign-tracking animals had enhanced CS-evoked phasic dopamine release during learning in comparison to control sign-tracking animals. Thus adolescent alcohol exposure promotes exaggerated sign-tracking responses mirrored by a potentiation in phasic dopamine signaling to incentive cues. Interestingly, after behavior had reached asymptote (last 25 trials, session 5), we found that phasic dopamine release was comparable between the treatment groups in response to CS presentation, supporting the view that dopamine's involvement is restricted to situations when conditions are changing and differential behavior is being acquired and established but not after stable responding has been achieved (Di Ciano et al, 2001; Clark et al, 2013). Indeed, significantly potentiated CS- and US-evoked phasic dopamine release in alcohol animals relative to controls during reacquisition mirrored the results from initial acquisition and supports the conclusion that animals exposed to alcohol in adolescence are particularly sensitive to fluctuating conditions and the attribution of updated incentive properties to reward-associated stimuli under those circumstances.

To further examine the hypothesis that alcohol-treated animals may be more responsive under conditions where outcomes are changing and deviating from expectation, all 
animals were given probe trials where reward size was varied unpredictably after behavior had reached asymptote. Consistent with previous reports (Hart et al, 2014; Tobler et al, 2005), phasic dopamine activity was modulated by reward size and expectation in both groups in a manner consistent with the reporting of a reward prediction error from formal models of reinforcement learning, with increased phasic dopamine signaling after better-than-expected outcomes and decreased phasic dopamine signaling after worse-thanexpected outcomes. Interestingly, our data showed that adolescent alcohol consumption promoted a greater sensitivity to the unexpected variation in reward sizes.

One potential limitation of the experiments outlined here is that animals in this study were singly housed during adolescence to permit accurate measures of voluntary alcohol intake, raising the question of whether or not there is an effect of housing condition in adolescence above and beyond that of alcohol exposure or whether the alcohol effects described here are dependent upon housing condition (Anderson et al, 2013). We cannot entirely rule out the possibility that housing conditions contribute to the overall pattern of conditioned responses reported here. However, control animals, housed in the identical conditions to that of the alcohol-exposed animals, show a distribution of conditioned responses that is consistent with previous work that systematically examined the population statistics of these behaviors (Fitzpatrick et al, 2013). This would suggest that singly housing animals in the current work did not shift the overall distribution of response biases and is therefore not the likely explanation for the extreme shift in the alcohol group exclusively to sign-tracking behavior. Importantly, a previous study in which rats were pair-housed and exposed to intragastric alcohol or control administration during adolescence observed that alcohol-treated animals showed increased lever-pressing behavior during Pavlovian conditioning consistent with the results outlined here (McClory and Spear, 2014), indicating that the effects of alcohol on learning are consistent across housing conditions. Finally, our work demonstrating that adolescent alcohol promotes maladaptive decision making (Nasrallah et al, 2011; Schindler et al, 2014), findings that we have previously linked to learning effects similar to the ones found here (Clark et al, 2012), have been replicated in animals that were group housed during intragastric alcohol administration in adolescence (Boutros et al, 2014). Thus the observed increase in sign-tracking behavior reported here is most likely the result of alcohol exposure rather than housing conditions. Indeed, this overall pattern of results suggests that the effects of adolescent alcohol exposure on the attribution of incentive value and decision making are quite robust as they have now been reported under multiple alcohol delivery paradigms (voluntary and involuntary) and with multiple housing conditions (single, paired, and group). A second potential limitation to the interpretation of the current results is the extent to which the findings are selective to alcohol exposure in adolescence. Previous studies have investigated the effect of nicotine and amphetamine exposure during adolescence on Pavlovian conditioned approach behavior in adulthood (Quick et al, 2014; Doremus-Fitzwater and Spear, 2011). Adolescent nicotine exposure (on PND 31-45) was shown to increase approach to a CS in male animals, whereas approaches to the CS were reduced in females. In addition, amphetamine sensitization during adolescence has also been shown to increase sign-tracking behavior. This raises the interesting possibility that an alteration to the assignment of incentive value to reward cues may be a general consequence of substance use in adolescence.

Collectively, these findings support theoretical accounts speculating that an imbalance in learning from positive and negative outcomes may be an important contributor to substance use disorders (Baker et al, 2011; Piray et al, 2010). Specifically, clinical research has demonstrated that the amount of alcohol consumed is reliably correlated with the degree to which individuals have positive over negative alcohol outcome expectancies (Jones et al, 2001). The current findings provide insight into the development of such an imbalance in the weighting of positive and negative experiences and a candidate neural mechanism underlying the neurocognitive and behavioral consequences of adolescent alcohol consumption that may contribute to an enhanced vulnerability for developing AUD in adulthood.

\section{FUNDING AND DISCLOSURE}

This work was supported by NIH grant R01AA021121 (to JJC) and the Utrecht University Short Stay PhD Fellowship (to MS). The authors declare no conflict of interest.

\section{ACKNOWLEDGMENTS}

We thank Scott Ng Evans for technical assistance and Shelly Flagel for assistance with data analysis.

\section{REFERENCES}

Alaux-Cantin S, Warnault V, Legastelois R, Botia B, Pierrefiche O, Vilpoux C et al (2013). Alcohol intoxications during adolescence increase motivation for alcohol in adult rats and induce neuroadaptations in the nucleus accumbens. Neuropharmacology 67: 521-531.

Anderson RI, Bush PC, Spear LP (2013). Environmental manipulations alter age differences in attribution of incentive salience to reward-paired cues. Behav Brain Res 257: 83-89.

Baker TE, Stockwell T, Barnes G, Holroyd CB (2011). Individual differences in substance dependence: at the intersection of brain, behaviour and cognition. Addict Biol 16: 458-466.

Bava S, Tapert SF (2010). Adolescent brain development and the risk for alcohol and other drug problems. Neuropsychol Rev 20: 398-413.

Bickel WK, Marsch LA (2001). Toward a behavioral economic understanding of drug dependence: delay discounting processes. Addiction 96: 73-86.

Blakemore SJ, Choudhury S (2006). Development of the adolescent brain: implications for executive function and social cognition. J Child Psychol Psychiatry 47: 296-312.

Blomeyer D, Friemel CM, Buchmann AF, Banaschewski T, Laucht M, Schneider M (2013). Impact of pubertal stage at first drink on adult drinking behavior. Alcohol Clin Exp Res 37: 1804-1811.

Boakes RA (1977). Performance on learning to associate a stimulus with positive reinforcement. In: Davis $\mathrm{H}$, Hurwitz $\mathrm{H}$ (eds). Operant-Pavlovian Interactions. Earlbaum: Hillsdale, NJ, USA, pp 67-97.

Boutros N, Semenova S, Liu W, Crews FT, Markou A (2014). Adolescent intermittent ethanol exposure is associated with increased risky choice and decreased dopaminergic and 
cholinergic neuron markers in adult rats. Int J Neuropsychopharmacol 18: pii: pyu003.

Casey BJ, Jones RM (2010). Neurobiology of the adolescent brain and behavior: implications for substance use disorders. J Am Acad Child Adolesc Psychiatry 49: 1189-1201; quiz 1285.

Chambers RA, Taylor JR, Potenza MN (2003). Developmental neurocircuitry of motivation in adolescence: a critical period of addiction vulnerability. Am J Psychiatry 160: 1041-1052.

Cheer JF, Wassum KM, Sombers LA, Heien ML, Ariansen JL, Aragona BJ et al (2007). Phasic dopamine release evoked by abused substances requires cannabinoid receptor activation. J Neurosci 27: 791-795.

Clark JJ, Collins AL, Sanford CA, Phillips PEM (2013). Dopamine encoding of pavlovian incentive stimuli diminishes with extended training. J Neurosci 33: 3526-3532.

Clark JJ, Nasrallah NA, Hart AS, Collins AL, Bernstein IL, Phillips PEM (2012). Altered risk-based decision making following adolescent alcohol use results from an imbalance in reinforcement learning in rats. PLoS One 7: e37357.

Clark JJ, Sandberg SG, Wanat MJ, Gan JO, Horne EA, Hart AS et al (2010). Chronic microsensors for longitudinal, subsecond dopamine detection in behaving animals. Nat Methods 7: 126-129.

Crews F, He J, Hodge C (2007). Adolescent cortical development: a critical period of vulnerability for addiction. Pharmacol Biochem Behav 86: 189-199.

Dawson DA, Goldstein RB, Chou SP, Ruan WJ, Grant BF (2008). Age at first drink and the first incidence of adult-onset DSM-IV alcohol use disorders. Alcohol Clin Exp Res 32: 2149-2160.

Day JJ, Roitman MF, Wightman RM, Carelli RM (2007). Associative learning mediates dynamic shifts in dopamine signaling in the nucleus accumbens. Nat Neurosci 10: 1020-1028.

Di Chiara G, Imperato A (1986). Preferential stimulation of dopamine release in the nucleus accumbens by opiates, alcohol, and barbiturates: studies with transcerebral dialysis in freely moving rats. Ann NY Acad Sci 473: 367-381.

Di Ciano P, Cardinal RN, Cowell RA, Little SJ, Everitt BJ (2001). Differential involvement of NMDA, AMPA/kainate, and dopamine receptors in the nucleus accumbens core in the acquisition and performance of pavlovian approach behavior. J Neurosci 21: 9471-9477.

Doremus-Fitzwater TL, Spear LP (2011). Amphetamine-induced incentive sensitization of sign-tracking behavior in adolescent and adult female rats. Behav Neurosci 125: 661-667.

Everitt BJ, Robbins TW (2005). Neural systems of reinforcement for drug addiction: from actions to habits to compulsion. Nat Neurosci 8: 1481-1489.

Fitzpatrick CJ, Gopalakrishnan S, Cogan ES, Yager LM, Meyer PJ, Lovic V et al (2013). Variation in the form of Pavlovian conditioned approach behavior among outbred male SpragueDawley rats from different vendors and colonies: sign-tracking vs. goal-tracking. PLoS One 10: e75042.

Flagel SB, Clark JJ, Robinson TE, Mayo L, Czuj A, Willuhn I et al (2011). A selective role for dopamine in stimulus-reward learning. Nature 469: 53-59.

Flagel SB, Robinson TE, Clark JJ, Clinton SM, Watson SJ, Seeman P et al (2010). An animal model of genetic vulnerability to behavioral disinhibition and responsiveness to reward-related cues: implications for addiction. Neuropsychopharmacology 35: 388-400.

Flagel SB, Watson SJ, Akil H, Robinson TE (2008). Individual differences in the attribution of incentive salience to a rewardrelated cue: influence of cocaine sensitization. Behav Brain Res 186: 48-56.

Goudriaan AE, Grekin ER, Sher KJ (2007). Decision making and binge drinking: a longitudinal study. Alcohol Clin Exp Res 31: 928-938.

Guerri C, Pascual M (2010). Mechanisms involved in the neurotoxic, cognitive, and neurobehavioral effects of alcohol consumption during adolescence. Alcohol 44: 15-26.
Hart AS, Rutledge RB, Glimcher PW, Phillips PE (2014). Phasic dopamine release in the rat nucleus accumbens symmetrically encodes a reward prediction error term. J Neurosci 34: 698-704.

Heien ML, Khan AS, Ariansen JL, Cheer JF, Phillips PE, Wassum KM et al (2005). Real-time measurement of dopamine fluctuations after cocaine in the brain of behaving rats. Proc Natl Acad Sci USA 102: 10023-10028.

Hingson RW, Heeren T, Winter MR (2006). Age at drinking onset and alcohol dependence: age at onset, duration, and severity. Arch Pediatr Adolesc Med 160: 739-746.

Johnson CA, Xiao L, Palmer P, Sun P, Wang Q, Wei Y et al (2008). Affective decision-making deficits, linked to a dysfunctional ventromedial prefrontal cortex, revealed in 10th grade Chinese adolescent binge drinkers. Neuropsychologia 46: 714-726.

Jones BT, Corbin W, Fromme K (2001). A review of expectancy theory and alcohol consumption. Addiction 96: 57-72.

Kelley AE (2004). Memory and addiction: shared neural circuitry and molecular mechanisms. Neuron 44: 161-179.

Lovic V, Saunders BT, Yager LM, Robinson TE (2011). Rats prone to attribute incentive salience to reward cues are also prone to impulsive action. Behav Brain Res 223: 255-261.

McClory AJ, Spear LP (2014). Effects of ethanol exposure during adolescence or in adulthood on Pavlovian conditioned approach in Sprague-Dawley rats. Alcohol 48: 755-763.

McMurray MS, Amodeo LR, Roitman JD (2014). Effects of voluntary alcohol intake on risk preference and behavioral flexibility during rat adolescence. PLoS One 9: e100697.

Meyer PJ, Lovic V, Saunders BT, Yager LM, Flagel SB, Morrow JD et al (2012). Quantifying individual variation in the propensity to attribute incentive salience to reward cues. PLoS One 7: e38987.

Milton AL, Everitt BJ (2012). The persistence of maladaptive memory: addiction, drug memories and anti-relapse treatments. Neurosci Biobehav Rev 36: 1119-1139.

Monti PM, Miranda R Jr, Nixon K, Sher KJ, Swartzwelder HS, Tapert SF et al (2005). Adolescence: booze, brains, and behavior. Alcohol Clin Exp Res 29: 207-220.

Nasrallah NA, Clark JJ, Collins AL, Akers CA, Phillips PE, Bernstein IL (2011). Risk preference following adolescent alcohol use is associated with corrupted encoding of costs but not rewards by mesolimbic dopamine. Proc Natl Acad Sci USA 108: 5466-5471.

Nasrallah NA, Yang TW, Bernstein IL (2009). Long-term risk preference and suboptimal decision making following adolescent alcohol use. Proc Natl Acad Sci USA 106: 17600-17604.

O'Brien CP, Childress AR, Ehrman R, Robbins SJ (1998). Conditioning factors in drug abuse: can they explain compulsion? J Psychopharmacol 12: 15-22.

Parkinson JA, Dalley JW, Cardinal RN, Bamford A, Fehnert B, Lachenal G et al (2002). Nucleus accumbens dopamine depletion impairs both acquisition and performance of appetitive Pavlovian approach behaviour: implications for mesoaccumbens dopamine function. Behav Brain Res 137: 149-163.

Paxinos G, Watson C (2004). The Rat Brain in Stereotaxic Coordinates - The New Coronal Set. Academic Press: Boston, MA, USA.

Philpot RM, Wecker L, Kirstein CL (2009). Repeated ethanol exposure during adolescence alters the developmental trajectory of dopaminergic output from the nucleus accumbens septi. Int $J$ Dev Neurosci 27: 805-815.

Piray P, Keramati MM, Dezfouli A, Lucas C, Mokri A (2010). Individual differences in nucleus accumbens dopamine receptors predict development of addiction-like behavior: a computational approach. Neural Comput 22: 2334-2368.

Quick SL, Olausson P, Addy NA, Taylor JR (2014). Repeated nicotine exposure during adolescence alters reward- related learning in male and female rats. Behav Brain Res 261: 171-176.

Robinson DL, Howard EC, McConnell S, Gonzales RA, Wightman RM (2009). Disparity between tonic and phasic ethanol-induced 
dopamine increases in the nucleus accumbens of rats. Alcohol Clin Exp Res 33: 1187-1196.

Robinson TE, Berridge KC (1993). The neural basis of drug craving - an incentive sensitization theory of addiction. Brain Res Rev 18: 247-291.

Robinson TE, Flagel SB (2009). Dissociating the predictive and incentive motivational properties of reward-related cues through the study of individual differences. Biol Psychiatry 65: 869-873.

Roitman MF, Stuber GD, Phillips PE, Wightman RM, Carelli RM (2004). Dopamine operates as a subsecond modulator of food seeking. J Neurosci 24: 1265-1271.

Rowland NE, Nasrallah N, Robertson KL. (2005). Accurate caloric compensation in rats for electively consumed ethanol-beer or ethanol-polycose mixtures. Pharmacol Biochem Behav 80: 109-114.

Salamone JD, Correa M (2012). The mysterious motivational functions of mesolimbic dopamine. Neuron 76: 470-485.

Saunders BT, Robinson TE (2010). A cocaine cue acts as an incentive stimulus in some but not others: implications for addiction. Biol Psychiatry 67: 730-736.

Saunders BT, Robinson TE (2012). The role of dopamine in the accumbens core in the expression of Pavlovian-conditioned responses. Eur J Neurosci 36: 2521-2532.

Saunders BT, Yager LM, Robinson TE (2013). Cue-evoked cocaine "craving": role of dopamine in the accumbens core. J Neurosci 33: 13989-14000.

Schindler AG, Tsutsui KT, Clark JJ (2014). Chronic alcohol intake during adolescence, but not adulthood, promotes persistent deficits in risk-based decision making. Alcohol Clin Exp Res 38: $1622-1629$.

Schultz W, Dayan P, Montague PR (1997). A neural substrate of prediction and reward. Science 275: 1593-1599.

Schultz W (2007). Multiple dopamine functions at different time courses. Annu Rev Neurosci 30: 259-288.

Shaham Y, Shalev U, Lu L, De Wit H, Stewart J (2003). The reinstatement model of drug relapse: history, methodology and major findings. Psychopharmacology (Berl) 168: 3-20.
Spear LP (2000). The adolescent brain and age-related behavioral manifestations. Neurosci Biobehav Rev 24: 417-463.

Squeglia LM, Pulido C, Wetherill RR, Jacobus J, Brown GG, Tapert SF (2012). Brain response to working memory over three years of adolescence: influence of initiating heavy drinking. J Stud Alcohol Drugs 73: 749-760.

Stewart J, de Wit H, Eikelboom R (1984). Role of unconditioned and conditioned drug effects in the self-administration of opiates and stimulants. Psychol Rev 91: 251-268.

Toalston JE, Deehan GA Jr, Hauser SR, Engleman EA, Bell RL, Murphy JM et al (2014). Reinforcing properties and neurochemical response of ethanol within the posterior ventral tegmental area are enhanced in adulthood by periadolescent ethanol consumption. J Pharmacol Exp Ther 351: 317-326.

Tobler PN, Fiorillo CD, Schultz W (2005). Adaptive coding of reward value by dopamine neurons. Science 307: 1642-1645.

Tomie A, Grimes KL, Pohorecky LA (2008). Behavioral characteristics and neurobiological substrates shared by Pavlovian signtracking and drug abuse. Brain Res Rev 58: 121-135.

Tomie A, Sharma N (2013). Pavlovian sign-tracking model of alcohol abuse. Curr Drug Abuse Rev 3: 201-219.

Verbeke G, Molenberghs G (2000). Linear Mixed Models for Longitudinal Data. Springer.

Witt ED (2010). Research on alcohol and adolescent brain development: opportunities and future directions. Alcohol 44: 119-124.

Yager LM, Pitchers KK, Flagel SB, Robinson TE (2014). Individual variation in the motivational and neurobiological effects of an opioid cue. Neuropsychopharmacology 40: 1269-1277.

Yager LM, Robinson TE (2013). A classically conditioned cocaine cue acquires greater control over motivated behavior in rats prone to attribute incentive salience to a food cue. Psychopharmacology (Berl) 226: 217-228.

Zeigler DW, Wang CC, Yoast RA, Dickinson BD, McCaffree MA, Robinowitz CB et al (2005). The neurocognitive effects of alcohol on adolescents and college students. Prev Med 40: 23-32. 